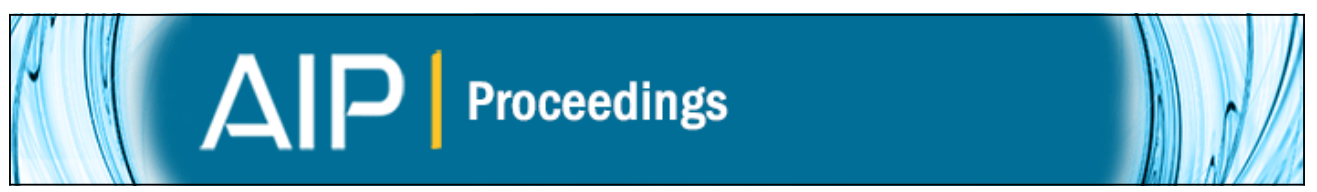

\title{
High Intensity Femtosecond XUV Pulse Interactions with Atomic Clusters
}

K. Hoffmann, B. Murphy, J. Keto, and T. Ditmire

Citation: AIP Conference Proceedings 1161, 270 (2009); doi: 10.1063/1.3241202

View online: http://dx.doi.org/10.1063/1.3241202

View Table of Contents:

http://scitation.aip.org/content/aip/proceeding/aipcp/1161?ver=pdfcov

Published by the AIP Publishing

\section{Articles you may be interested in}

High-order harmonic generation during propagation of femtosecond pulses through the laserproduced plasmas of semiconductors

J. Appl. Phys. 117, 023114 (2015); 10.1063/1.4905902

Application of carbon cluster-contained extended plasmas for the high-order harmonic generation of ultrashort pulses

J. Appl. Phys. 115, 183101 (2014); 10.1063/1.4875681

Ultrahigh Intensity Laser-Matter Interaction Studies at RRCAT, Indore AIP Conf. Proc. 1308, 51 (2010); 10.1063/1.3526163

Plasma mirror distortions and parametric instabilities induced by high intensity femtosecond pulses on solid targets

AIP Conf. Proc. 611, 294 (2002); 10.1063/1.1470315

High intensity 30 femtosecond laser pulse interaction with thin foils

AIP Conf. Proc. 426, 253 (1998); 10.1063/1.55231 


\title{
High Intensity Femtosecond XUV Pulse Interactions with Atomic Clusters
}

\author{
K. Hoffmann, B. Murphy, J. Keto, and T. Ditmire \\ The Texas Center for High Intensity Laser Science \\ Department of Physics \\ University of Texas at Austin, MS CI 600, Austin, TX 78712 \\ Phone: 512-471-3296
}

\begin{abstract}
The interactions of large xenon clusters irradiated by intense, femtosecond extremeultraviolet pulses at a wavelength of $38 \mathrm{~nm}$ have been studied. Using high harmonic generation from a $35 \mathrm{fs}$ near-infrared terawatt laser, clusters have been irradiated by XUV pulses of $10^{11}$ $\mathrm{W} / \mathrm{cm}^{2}$ intensity. Charge states up to $\mathrm{Xe}^{8+}$ are observed, states well above that produced by single atom illumination, indicating that plasma continuum lowering is important. Furthermore the kinetic energy distribution of the exploding ions is consistent with a quasineutral hydrodynamic expansion, rather than a Coulomb explosion.
\end{abstract}

Keywords: Intense Lasers, Higher Harmonic Generation, Rare Gas Clusters

PACS: $36.40 . \mathrm{Gk}, 36.40 . \mathrm{Qv}, 52.50$

\section{INTRODUCTION}

The nature of the interactions between high-intensity, ultrafast, near-infrared laser pulses and atomic clusters of a few hundred to a few hundred thousand atoms has been well studied over the past few years by a number of groups world wide [1-3]. Such studies have found some rather unexpected results, including the striking finding that these interactions appear to be more energetic than interactions with either single atoms or solid-density plasmas. The reason is that atoms in clusters can absorb large amounts of energy from the laser field, producing $\mathrm{x}$ rays, hot electrons and energetic ions in a process dominated by the large ponderomotive force [1]. At high intensity, the electric field of the laser strips electrons from the cluster by tunnel and barrier suppression ionization, and electrons confined to the vicinity of the cluster by spacecharge forces further ionize the cluster to high charge states by collisional ionization [1]. If the laser field is strong enough, a condition satisfied if the ponderomotive potential of the laser is greater than the surface binding potential of the ionized cluster, the laser field strips the cluster of its electrons and the charged cluster explodes by Coulomb explosion [4]. On the other hand, if the clusters are large and the ions highly charged, space charge forces retain electrons within the cluster body, resulting in a "nanoplasma" which explodes by hydrodynamic forces [1].

Recently, the interactions of intense, extreme ultraviolet (XUV) pulses (of $10-100$ $\mathrm{nm}$ wavelength) with atomic clusters have been explored by a number of groups [513]. The time scale and mechanisms of the explosion of these clusters in the intense 
XUV pulse is an open question, and such interactions are expected to be dramatically different than experiments with intense IR laser pulses. Electrons are easily liberated initially by single photon ionization which can sequentially strip the constituent atoms to high charge states, however subsequently ionized electrons are not easily removed from the cluster's space-charge forces because of the weak ponderomotive potential of the short wavelength pulse. It is, therefore, not clear that a nanoplasma will form as the XUV pulse ejects photoionized electrons sequentially, and it is open question whether XUV irradiated clusters will explode by Coulomb explosion or by hydrodynamic forces.

The first experiments on intense VUV irradiated clusters have been performed at the DESY VUV-FEL 2002 with Xe clusters of sizes ranging up to $\sim 30,000$ atoms per cluster [5-8]. This group observed the surprising result that energetic electrons and high ion charge states $\left(\mathrm{Xe}^{8+}\right)$ are produced when clusters are irradiated with $95 \mathrm{~nm}$ light at intensities of $\sim 10^{13} \mathrm{~W} / \mathrm{cm}^{2}[5]$ and that the mechanism for ejecting the high Xe charge states from the clusters was by a Coulomb explosion. Later experiments at 95 $\mathrm{nm}$ showed electron emission from moderate sized ( 1000 atom) $\mathrm{Ar}$ and Xe clusters with a quasi-Maxwellian distribution with a $9 \mathrm{eV}$ electron temperature [7]. More recent experiments at $33 \mathrm{~nm}$ in small $(<100$ atom) clusters have also been reported, with a different conclusion [8]. In those experiments at shorter wavelength the electron removal from the cluster was purely sequential, and that authors saw little evidence for the production of a nanoplasma or a thermal electron distribution [8].

These experiments have generated a number of theoretical studies, focused in particular on the reasons for the high-charge-state production and anomalously high absorption seen in the DESY experiments. At present there have been at least three distinct models advanced to explain the DESY results [10-12]. Santra and Greene have proposed that the large absorption of VUV irradiated clusters and high charge states observed can be explained by a thermalized nanoplasma heated by inverse bremsstrahlung absorption with appropriately corrected collision rates [10]. Siedschlag and Rost have posited that the high charge states are produced by single photon absorption in the cluster facilitated by the distortion of the ionic binding potential by the neighboring ions in the cluster [11]. Finally, Jungreuthmayer et al. have performed simulations which indicate that the high charge states result from collisional ionization by an electron cloud heated through a process they refer to as many-body recombination heating [12]. The common denominator of most of these models is that a cluster nanoplasma is formed, a conclusion not completely supported by data, which have suggested a Coulomb explosion $[5,8]$ is the main explosion mechanism.

We have been conducting first laser based studies by converting light from a highenergy femtosecond laser to the short wavelength region through high-order-harmonic generation [13]. These harmonics are focused up to $10^{11} \mathrm{~W} / \mathrm{cm}^{2}$ into a cluster jet and the ions and electrons ejected are analyzed by time-of-flight methods. Our first experiments have been performed on Xe clusters irradiated by intense $32.6 \mathrm{eV}$ pulses. Very similar to the DESY experiment we observed surprisingly high charge states from the Xe clusters but with lower kinetic energies. The directly measured kineticenergy spectra showed that the clusters explode rather by hydrodynamic forces with an electron temperature of $8 \mathrm{eV}$ [13]. 


\section{EXPERIMENTAL PROCEDURE}

In our studies, we employed intense pulses of XUV light produced by high harmonic generation (HHG) of an intense femtosecond laser and refocused these pulses into a cluster-producing gas jet. Our basic experimental layout is illustrated in figure 1 .

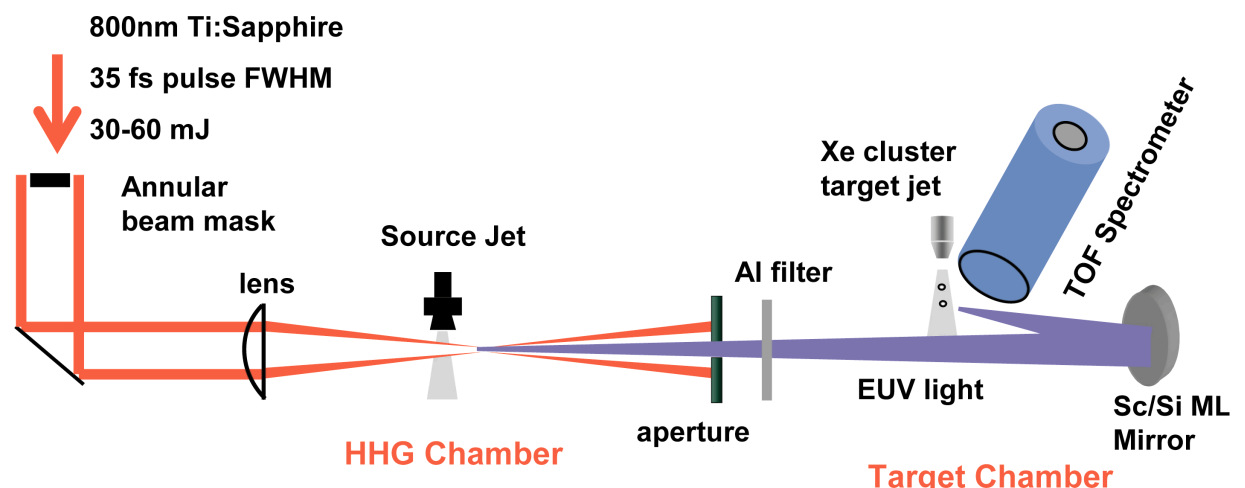

FIGURE 1. Illustration of the high harmonic producing chamber and XUV cluster target chamber. Here pulses of $38 \mathrm{~nm}$ light produced by harmonic generation with $35 \mathrm{fs}, 800 \mathrm{~nm}$ pulses are isolated and refocused in a secondary chamber.

The output of the THOR laser, a CPA Ti:sapphire system delivering $800 \mathrm{~nm}, 35 \mathrm{fs}$ pulses of energy up to $500 \mathrm{~mJ}$, was loosely focused into a pulsed jet of 200 psi argon with a $2 \mathrm{~m}$ focal length quartz lens (f/60) to an intensity of $\sim 10^{15} \mathrm{~W} / \mathrm{cm}^{2}$. Higher harmonic production was optimized by positioning the jet directly above the laser beam and $150 \mathrm{~mm}$ upstream of the focus. A mask placed 4 meters upstream of the lens obscured the central portion of the infrared pulse, and this mask was imaged to an iris 4 meters after the lens to remove most of the infrared light. The near Gaussian, diffraction limited HHG light is more collimated and passes through the iris [14]. A $200 \mathrm{~nm}$ thick Al filter (Luxel Corp.) blocked any residual infrared light from reaching the XUV interaction chamber. After separating the infrared from the XUV harmonics, we select and focus the $21^{\text {st }}$ harmonic using a spherical $\mathrm{Sc} / \mathrm{Si}$ multilayer mirror designed to reflect light at $38 \mathrm{~nm} \pm 5 \mathrm{~nm}$ with $\mathrm{f} / 10$ geometry at near normal incidence [15].

To measure the entire harmonic spectrum we replaced the multilayer mirror with a flat-field grazing-incidence XUV spectrograph. The design is based on a mechanically ruled aberration-corrected concave grating [16] dispersing the higher harmonics on a MCP-Phosphor screen detector. Figure 2a shows a typical line out of the plateau and cutoff of harmonics in argon.

To measure the energy and spatial profile of the focused $21^{\text {st }}$ harmonic, we positioned an XUV sensitive photodiode (IRD XUV-10) after the focus and scanned a knife edge perpendicular to the beam axis on different positions. The data for the smallest focus size are illustrated in figure $2 \mathrm{~b}$. We can fit an $8 \mu \mathrm{m}$ focal spot diameter $\left(1 / \mathrm{e}^{2}\right.$ full width) to the derivative at an average total energy of $0.5 \mathrm{~nJ}$. Assuming 10-15 
fs duration for the HHG pulse due to ionization of the argon, this yields a focal intensity of $\sim 10^{11} \mathrm{~W} / \mathrm{cm}^{2}$.
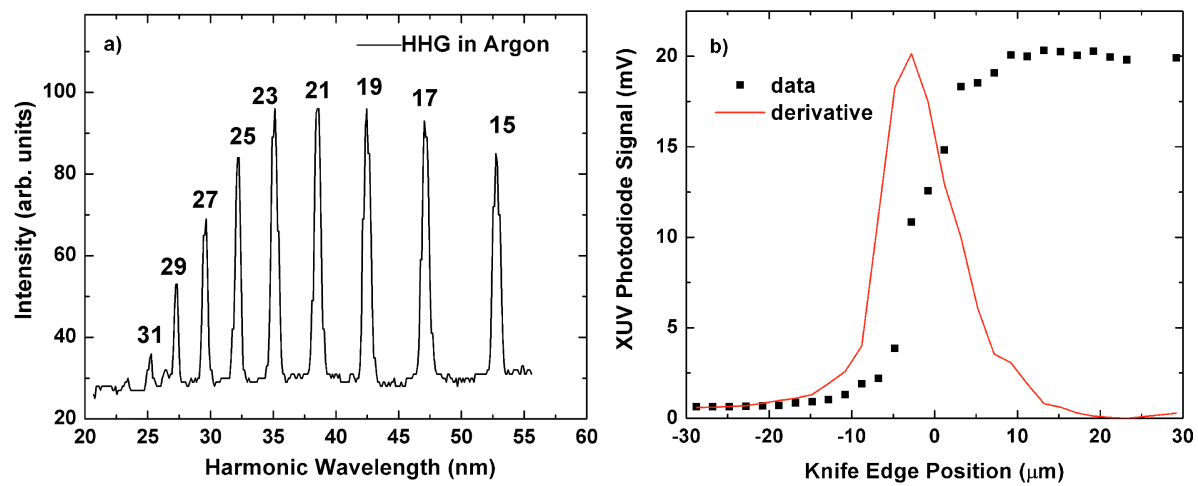

FIGURE 2. a) Higher harmonics dispersed in a XUV Spectrograph on a MCP-Phosphor Screen Detector. b) Measured signal in an XUV sensitive photodiode at the XUV pulse focus passing around a razor blade as a function of blade position in the focus. The solid line shows the derivative and fits a Gaussian focus of an $8 \mu \mathrm{m}\left(1 / \mathrm{e}^{2}\right)$ diameter.

A second pulsed gas jet nozzle mounted directly above the XUV focus produced a jet of xenon clusters. This jet, with a throat diameter of $500 \mu \mathrm{m}$, was backed with a pressure of 13 bar. Using previously published results to ascertain the average size and size distribution of the Xe clusters produced by this jet we estimated that we produced a log-normal distribution of clusters with average size, $<\mathrm{N}>$, ranging up to $\approx 10^{4}$ atoms [17]. A Wiley-McLaren [18] time-of-flight (TOF) spectrometer with charged grids mounted around the XUV interaction region allowed us to measure the ion charge state distribution. Extracted ions traveled through a field-free region to a multichannel plate, whose output is recorded on an oscilloscope or time-delay-counting card. When the extraction grids were grounded, the energetic ions from the explosions of the clusters traveled by their own initial kinetic energy to the MCP detector. In this way, a Xe ion energy spectrum could be recovered from the Xe ion time of flight.

\section{RESULTS AND DISCUSSION}

Figure 3 shows the TOF spectrum from the Xe jet produced at various backing pressures when irradiated with the $21^{\text {st }}$ harmonic at constant intensity. High charge states appear at backing pressures where clustering begins. Multiple XUV photon absorption occurs even in the case of monomer irradiation, resulting in $\mathrm{Xe}^{3+}$ production (with some possible evidence for $\mathrm{Xe}^{4+}$ which would have to result from two photon ionization). There is a small contribution of $\mathrm{Ar}^{+}$ions from the $\mathrm{HHG}$ chamber overlapping with the the $\mathrm{Xe}^{3+}$ peak. Higher charge states are produced when clusters begin to form in the jet. We observe significant number of ions with charge states up to $5+$ when the cluster size distribution has average cluster size $\geq 1500$ atoms per cluster. Furthermore, we observe a small number of ions with charge states between $6+$ and $8+$. At the largest cluster sizes the peaks broaden and smear out due to kinetic energy. Although the abundance of highly charged ions is lower in our data 
than in DESY results at pulse intensity nearly two orders of magnitude higher, we see the onset of highly charged ions in the same cluster-size regime.

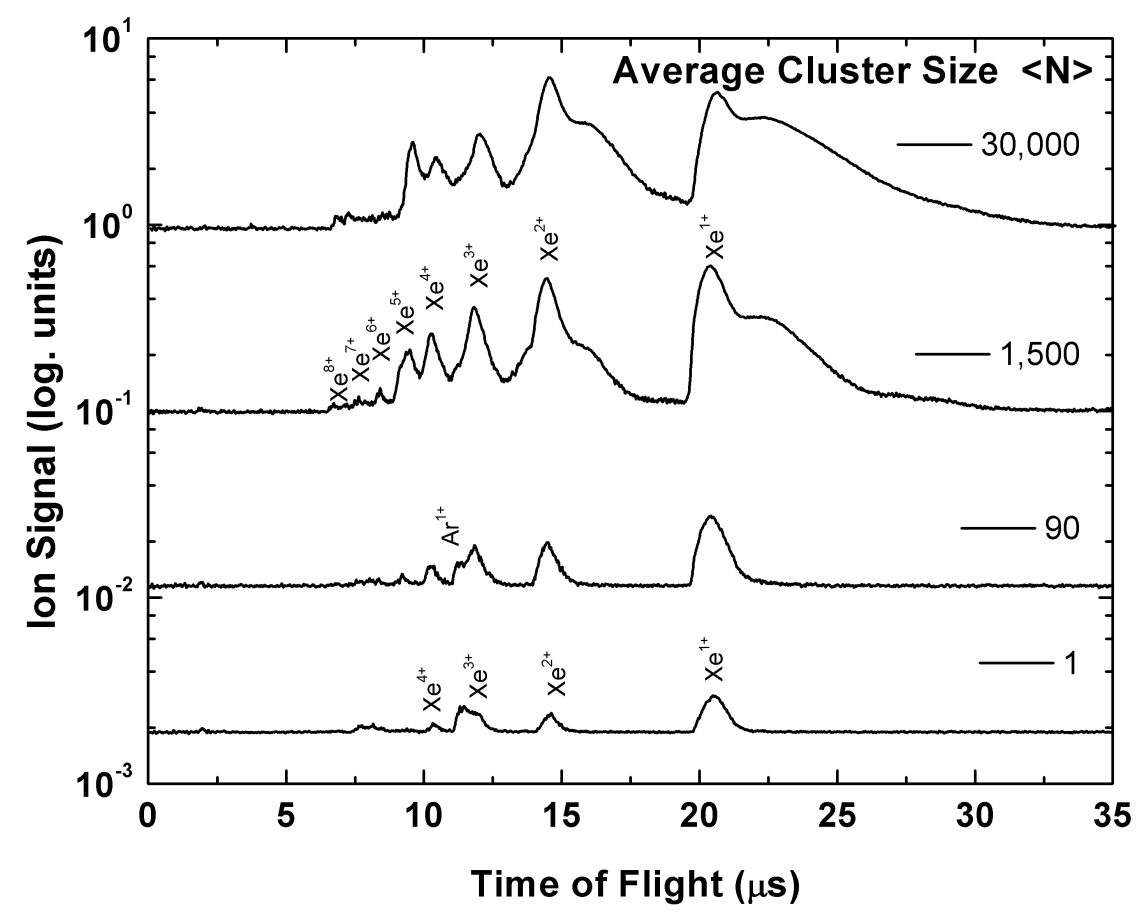

FIGURE 3. Ion charge state time-of-flight spectra for various xenon cluster sizes. The intensity is held constant at $1 \times 10^{11} \mathrm{~W} / \mathrm{cm}^{2}$.

A broadening of the TOF peaks occurs at large cluster sizes, though we do not believe that this broadening results from kinetic energy of the ions ejected by exploding clusters, which was the explanation assigned to broadening observed in the DESY Xe cluster experiments [5]. We followed the DESY approach [6] and performed Monte Carlo style simulations of ion trajectories originating from Coulomb explosions. Isotropic ion distributions with a Gaussian energy profile were used as input parameters of the SIMION code [19], representing our geometric setup, to simulate TOF spectra (a Maxwellian distribution would fit a hydrodynamic expansion better). A typical TOF setup is shown schematically in figure $4 \mathrm{a}$. After an isotropic cluster explosion only forward and backward ejected ions will reach the detector. All other ions will hit the grid assembly or the chamber walls. This gives rise to a very characteristic peak shape at the detector. The trace in figure 4a shows a simulation for xenon ions with $100 \mathrm{eV}$ mean-kinetic-energy distribution and a width of $100 \mathrm{eV}$. Low kinetic energy ions will get focused by the TOF to a center peak, forward ejected ions appear in a peak at earlier arrival times and backward ejected ions in a peak with a sharp cutoff, due to the repelling potential of the back grid, at later times. 

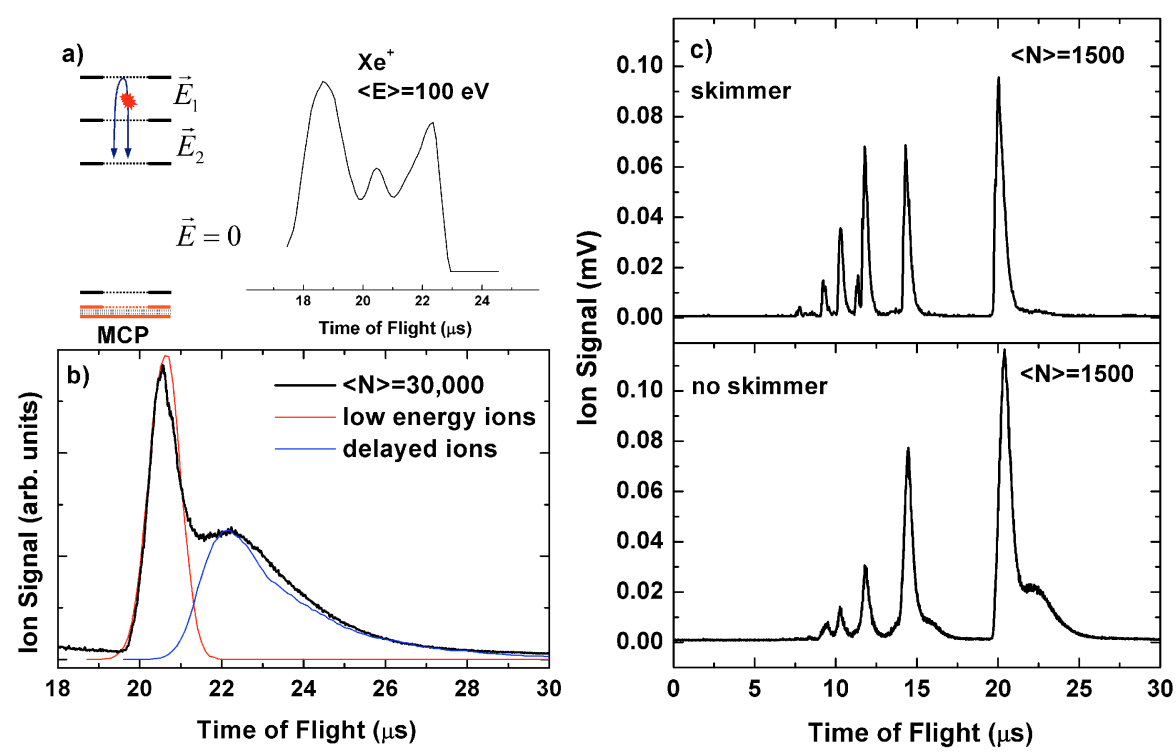

FIGURE 4. a) Typical TOF setup, only forward and backward ejected ions from isotropic explosions reach the detector, this gives rise to a characteristic peak shape. b) A delayed charge transfer process to the background gas is responsible for the peak broadening. c) By introducing a skimmer we are able to reduce the background gas and thus the charge transfer contribution.

We could not reproduce the measured $\mathrm{Xe}^{+}$peak shape shown in figure $4 \mathrm{~b}$ by this procedure. Instead, we attribute this peak broadening, which manifests itself as a growing feature only on the slow side of the $\mathrm{Xe}^{+}$peak, with ions produced by a charge transfer process between extracted ions from the interaction region on their way to the detector with neutral surrounding Xe atoms [20]. Assuming a relatively low kinetic energy of $8 \mathrm{eV}$ for the $\mathrm{Xe}^{+}$ions we were able to fit the first peak. These ions are now accelerated by the TOF in the E1 and E2 field region. Here they undergo a charge transfer reaction (according to Eq. 1) and generate secondary ions, which then are accelerated by the TOF field to the detector. This gives rise to the second delayed peak structure. This process is very well studied and described over the past 50 years in literature $[20,21]$. It follows the general schema:

$$
A^{q+}(f \text { as })+A(\text { thermal }) \rightarrow A(f \text { as })+A^{q+}(\text { near - thermal })
$$

and two specific major pathways for Xe ions in our experiment:

$$
\begin{aligned}
& X e^{+}+X e \rightarrow X e+X e^{+} \\
& X e^{2+}+X e \rightarrow X e+X e^{2+} \rightarrow X e^{+}+X e^{+}
\end{aligned}
$$


To prove our hypothesis we introduced differential pumping and skimmed the cluster beam. This greatly reduced the background pressure and with that the charge transfer process figure $4 \mathrm{c}$. Introducing the skimmer (upper picture) narrows the peaks and enhances the overall TOF resolution.

The next question that we wished to address was whether these highly charged ions in the cluster exploded by a Coulomb explosion, as has been surmised in the DESY results, both at $100 \mathrm{~nm}$ and $30 \mathrm{~nm}$. To do this we examined the energy spectrum from the exploding Xe clusters by field-free time of flight by the ejected ions. Figure 5 shows the energy spectrum of Xe ions ejected from the exploding clusters when the average cluster size is 30,000 atoms. This ion distribution peaks at around $80 \mathrm{eV}$ and has a tail that extends out toward $600 \mathrm{eV}$.

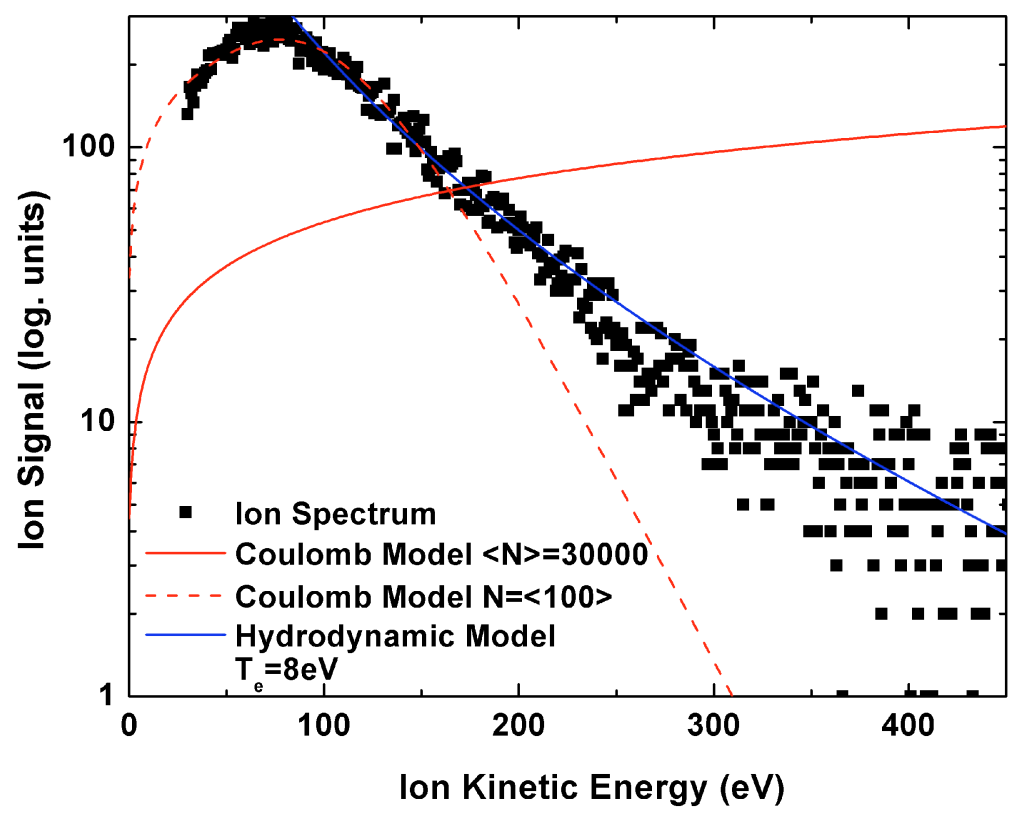

FIGURE 5. Xenon ion kinetic energy spectrum from field-free time-of-flight of a exploding cluster ion $<\mathrm{N}>=30,000$ at $1 \times 10^{11} \mathrm{~W} / \mathrm{cm}^{2}$. The spectrum is fit to a model of Coulomb explosion of large clusters (red solid line) and small clusters (dashed line), as well as the spectrum of a quasineutral expansion of a spherical cluster with an initial temperature of $8 \mathrm{eV}$ (blue solid line).

We can calculate the spectrum from Coulomb exploding clusters by convolving the spectrum of a single Coulomb exploding cluster with a log-normal cluster size distribution, a procedure which has been shown to model accurately the explosions of deuterium clusters irradiated by high intensity $800 \mathrm{~nm}$ laser pulses [4]. The calculated spectrum for Coulomb exploding clusters with $<\mathrm{N}>=30,000$ (and $\mathrm{Z} \sim 1$ ) is illustrated in figure 5. This distribution is very different than the observed spectrum with a peak well above $2 \mathrm{keV}$. For comparison, the spectrum from a best fit distribution with $<\mathrm{N}>$ $=100$ is also illustrated in this figure. Such a Coulomb explosion distribution accurately matches the shape of the ion spectrum peak at $100 \mathrm{eV}$ but does not accurately match the tail of the energy distribution. On the other hand, we have fitted 
our observed ion spectrum to that of a hydrodynamically expanding quasi-neutral cluster nanoplasma [22]. This best fit passes through the data over the entire energy range from $10 \mathrm{eV}$ to $600 \mathrm{eV}$. This spectrum is consistent with an initial electron temperature in the $\mathrm{Xe}$ cluster plasmas of $8 \mathrm{eV}$. (This electron temperature is very close to that of the electron spectrum observed in ref. [7] from XUV FEL irradiation of Xe clusters.)

The observed ion energy spectrum is strong evidence that near quasi-neutral nanoplasmas are formed in our XUV pulse irradiation of the Xe clusters and that the explosion mechanism is not by Coulomb explosion but instead by hydrodynamic expansion. A temperature of $8 \mathrm{eV}$ is consistent with the amount of heating we expect by inverse bremsstrahlung (IB) in the XUV field, which we have estimated by calculating the electron heating using the bremstrahlung absorption rate for high energy photons [23]. More enigmatic is the production of high charge states under these conditions. The models of both Jungreuthmayer et al. [12] and Santra and Greene [10] propose that the high charge states observed in high intensity XUV irradiation of clusters results from electron collisional ionization of ions in the transient nanoplasma that is formed in the cluster. Such a process is well documented in IR irradiated clusters. The same model has been further explored by Ziaja et al. [24]. However, this picture is inconsistent with our data. The equilibrium charge state distribution found by numerical solution of the Saha equations for this density and 8 $\mathrm{eV}$ temperature is $\mathrm{Z} \sim 2.4$. The calculated charge state distribution has no significant number of ions with charge greater than $3+$. It seems that collisional ionization alone cannot explain our observations.

The model of Siedschlag and Rost, however, suggests that the high charge states result from the distortion of the ionic binding potential by neighboring charged ions [11]. Strictly speaking, this picture is inaccurate in a quasi-neutral plasma, like that which persists in our clusters, because the electrons will tend to shield the fields of the neighboring ions. In a later paper they point out that electron screening is important in determining the extent of the lowering of the ionization potential of ions in the cluster nanoplasma [25]. We believe that our data, which appear to arise from explosions of nearly quasineutral clusters, can be explained within the scope of this picture using a simple ionization potential lowering model. In a dense plasma the presence of an electron cloud around an ion will lower the ionization potential (even when the plasma is effectively neutral) because of the reduced energy needed to liberate an electron into the sea of electrons around the plasma's ions, an effect commonly termed continuum lowering. In our strongly coupled cluster nanoplasmas (where the strong coupling parameter $\Gamma \sim 0.7$ ), the usual Debye-Hueckel model is not appropriate [26,27], but we can use the ion-sphere model [26] to calculate the magnitude of this continuum lowering in our Xe nanoplasmas. In this regime the ionization potential will be lowered by

$$
\Delta I P=9 / 10(2 \overline{\mathrm{Z}}-1) \mathrm{e}^{2} \mathrm{n}_{\mathrm{i}}^{1 / 3} / 4 \pi \varepsilon_{0},
$$

where $\mathbf{n}_{\mathrm{i}}$ is the ion density in the cluster. This predicts that at the ion density of the unexpanded cluster, which has been ionized to $Z \sim 4$, the ionization potential will be lowered by about $24 \mathrm{eV}$. This would be sufficient to reduce the ionization potential of $\mathrm{Xe}^{5+}$ sufficiently (where IP $\sim 54 \mathrm{eV}$ ) to allow direct photo-ionization from the $\mathrm{Xe}^{4+}$ (as 
well as the lower charge states up to this point). An estimate of the photoionization cross section of the lowered potential of $\mathrm{Xe}^{5+}$ indicates that a high degree of photoionization is possible given the photon fluence of our XUV pulse. This plasma continuum lowering effect will be at work in a completely quasineutral cluster plasma, so it is consistent with the observation of an ion energy spectrum that appears to arise from the hydrodynamic expansion of quasineutral cluster nanoplasmas.

The presence of a small number of charge states with $Z=6-8+$ can then be explained by nonequilibrium collisional ionization of the $\mathrm{Xe}^{5+}$ ions produced rapidly ( $\sim 20 \mathrm{fs}$ ) by photoionization during the XUV pulse by the hot tail of the $8 \mathrm{eV}$ electron distribution. Using the usual Lotz formula for the collisional ionization cross sections [28] with ionization potentials corrected by continuum lowering and integrating this cross section over an $8 \mathrm{eV}$ Maxwellian, we calculate that roughly $5 \%$ of $\mathrm{Xe}^{5+}$ ions can be collisionally stripped up to $\mathrm{Xe}^{8+}$ during $\sim 1.5 \mathrm{ps}$, the time for a $15 \mathrm{~nm}, 8 \mathrm{eV}$ cluster to expand hydrodynamically to roughly twice the initial radius. So the presence of small numbers of $\mathrm{Xe}^{6+}-\mathrm{Xe}^{8+}$ in our data can be explained by electron collisional ionization from the photoionized $\mathrm{Xe}^{5+}$ state. The charge states are subsequently frozen in place by the subsequent expansion of the cluster, an effect well known in cluster explosion experiments driven by IR radiation [2,3]. We have also estimated the probability of two-photon ionization of $\mathrm{Xe}^{5+}$ to these higher charge states and have concluded that the intensity of our XUV pulse is well below that needed to lead to significant ionization to $\mathrm{Xe}^{6+}-\mathrm{Xe}^{8+}$.

In summary, we have experimentally studied the explosions of large Xe clusters when irradiated by intense, femtosecond pulses of XUV radiation produced by high order harmonic conversion of a Ti:sapphire laser. We find that the ion spectrum of the explosion is consistent with that of the expansion of a quasineutral plasma with electron temperature of $8 \mathrm{eV}$ and inconsistent with a Coulomb explosion. Charge states up to $5+$ observed in these explosions are produced by photoionization of ions whose ionization potentials have been depressed by plasma continuum lowering and a small fraction of ions are further stripped by nonequilibrium electron collisional ionization.

\section{ACKNOWLEDGMENTS}

This work was supported by the DOE Office of Basic Energy Sciences and the National Nuclear Security Administration under Cooperative agreement DE-FC5203NA00156.

\section{REFERENCES}

1. T. Ditmire et al. Phys. Rev. A. 53, 3379 (1996).

2. M. Lezius et al., Phys. Rev. Lett. 80, 261 (1998).

3. T. Ditmire et al., Nature (London) 386, 54 (1997)

4. K.W. Madison et al., Phys. Plasmas 11, 270 (2004).

5. H. Wabnitz et al. Nature 420, 482 (2002).

6. T. Laarmann, et al., Phys. Rev. Lett. 92, 143401 (2004).

7. T. Laarmann et al., Phys. Rev. Lett. 95, 063402 (2005).

8. C. Bostedt et al., Phys. Rev. Lett. 100, 133401 (2008). 
9. S. Namba et al. Phys. Rev. Lett. 99, 043004 (2007)

10. R. Santra and C. H. Greene, Phys. Rev. Lett. 91, 233401 (2003).

11.C. Siedschlag and J-M Rost, Phys. Rev. Lett. 93, 043402 (2004).

12.C. Jungreuthmayer, et al, J. Phys. B: At. Mol. Opt. Phys. 38, 3029 (2005).

13. B. Murphy et al, Phys. Rev. Lett. 101, 203401 (2008).

14. J. Peatross et al., Opt. Lett. 19, 942 (1994).

15. Y. A. Uspenskii et al., Opt. Lett. 23, 771 (1998).

16. T Kita et al, Appl. Optics 22, 4, 512 (1983)

17. F. Dorchies et al., Phys. Rev. A 68, 023201 (2003).

18. W.C. Wiley, I.H. MacLaren, Rev. Sci. Instr., 26, 1150 (1955)

19. D.A. Dahl, SIMION 3D Version 6.0 Ion Optics Simulation Software

20. J. B. Hasted and M. Hussain, Proc. Phys. Soc. London 83, 911 (1964).

21. J.S. Miller et al, J. of Appl. Phys. 91, 984, (2002)

22. R. F. Schmalz, Phys. Fluids 28, 2923 (1985).

23. Ya.B. Zel'dovich and Yu. P. Raizer, Physics of Shock Waves and High Temperature Hydrodynamic Phenomena (Dover, Mineola, NY, 2002), p. 259.

24. B. Ziaja, H. Wabnitz, E. Weckert, and T. Moeller, Europhys. Lett. 82, 24002 (2008).

25. I. Georgescu, U. Saalmann, and J. M. Rost, Phys. Rev. A 76, 043203 (2007).

26. R. P. Drake, High Energy Density Physics: Fundamentals, Inertial Fusion and Experimental Astrophysics (Springer Verlag, Berlin, 2006), p. 73.

27. G. Ecker and W. Kroell, Phys. Fluids 6, 62 (1963).

28. W. Lotz, Z. Phys. 206, 205 (1967). 